\title{
Electrospinning SA@PVDF-HFP Core-Shell Nanofibers Based on Visual Light Transmission Response to Alcohol for Intelligent Packaging
}

Yanan Xiao ${ }^{1}$, Fengwei Xie ${ }^{2 *}$, Hao Luo ${ }^{1}$, Rongxing Tang ${ }^{1}$ and Jiazi Hou ${ }^{1 *}$

${ }^{1}$ Key Laboratory of Automobile Materials, Ministry Education, School of Materials Science and Engineering, Jilin University, Changchun 130025, China

${ }^{2}$ School of Engineering, Newcastle University, Newcastle upon Tyne, NE1 7RU, United Kingdom

KEYWORDS: electrospinning; fibrous membrane; core-shell structure; light transmission response; alcohol detection;

ABSTRACT: A naked-eye detector based on a rapid transmittance response to alcohol was designed to offer real-time and reusable detection of fruit freshness. To ensure the hydrophobicity of the fibrous membrane and high light transmission response to alcohol, fluorine-rich poly(vinylidene fluoride-co-hexafluoropropylene) (PVDF-HFP) with low refractive index was selected as the shell layer while sodium alginate (SA) and polyvinyl alcohol (PVA) were selected as the core layer for coaxial electrospinning. The core-shell fibrous detector was obtained by treatment with $\mathrm{CaCl}_{2}$ to form a stable 
hydrogel and by water flushing to remove PVA. The interior structure of the fiber and its evolution were investigated with increasing SA concentration, which changed from a non-concentric structure to a core-shell structure. Without SA, non-concentric structured fibers were obtained due to high flowability and incompatibility between the organic solvent phase of PVDF-HFP and the aqueous phase of PVA. As the SA concentration increased, the enhanced viscosity and surface tension decreased the asymmetric mobility significantly, which competed with the charge attractive forces from the Taylor cone surface, leading to a core-shell structure. The as-spun membranes were opaque due to light scattering at the interface between air and fiber, and became light transparent after immersion in a rotten fruit containing alcohol and acetic acid due to a decreased light loss. The rapidly responsive, reusable fibrous membranes with over $90 \%$ light transparency developed here have high potential for application in visual intelligent packaging to monitor the freshness of fruits and vegetables.

\section{Introduction}

Rapidly responsive and simple intelligent packaging to monitor the freshness of fruits is highly desired in response to the explosive growth of food corruption caused by improper food storage, which is common nowadays. Foods may be under improper or prolonged storage conditions, and ordinary packaging prevents the spoilage from being readily observed. However, packaging can block oxygen and water, which is necessary for microbial growth, and without it, the speed of spoilage would be accelerated. 
Consumption of spoiled vegetables and fruits may lead to food poisoning, causing gastrointestinal discomfort, fever, and even death. In this scenario, intelligent packaging that can instantaneously and visually monitor the freshness of fruits and vegetables is highly necessary. During the past several years, various food freshness detection strategies were proposed, such as gas ${ }^{1-3}, \mathrm{pH}^{4-5}$, light, and amperometric responsiveness ${ }^{6-7}$. Among these strategies, detection of the rotten products of food to understand food corruption provides an effective solution. In this regard, artificial scent screening systems such as electronic noses or E-noses are popular. Based on chemical adsorption methods, such systems can monitor food freshness by the reaction of halochromic dyes in a cross-reactive colorimetric barcode with spoilage product gases ${ }^{8-9}$. Zhang et al. ${ }^{10}$ fabricated amine-responsive fluorescent materials comprised of multiple porous nanocomposites of chitosan, dye, and cellulose acetate to detect the shrimp and crab freshness in real-time by identifying the amine generated during food spoilage. Recently, Bianchi et al. ${ }^{11}$ reported an indicator label identifying the freshness by a multilayer $\mathrm{pH}$-sensitive composite such as cellulose acetate and black carrot anthocyanin. Unfortunately, this smart packaging based on the irreversible discoloration of the indicator owing to the time and temperature changes in the storage and transportation process, could not be reused ${ }^{12-13}$. Considering that alcohol is the main rotten product of fruit with high sugar content, the amount of alcohol produced directly reflect the degree of decay and thus the quantitative detection of the alcohol provides another effective solution to monitor the freshness ${ }^{14}$. Usually, the alcohol 
concentration is related to a change in relative resistance, conductance, or current under specified environmental conditions ${ }^{15}$. Up to now, some studies based on the above-

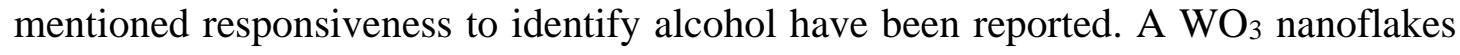
sensor for selective ethanol sensing was fabricated by Spagnoli et al. ${ }^{16}$. The $\mathrm{WO}_{3}$ was screen-printed as a paste on an alumina substrate, which responded well to alcohol and the conductance remained constant in mild humidity conditions. A sensor composed of Mo-doped $\mathrm{SnO}_{2}$ nanotubes also exhibited excellent alcohol sensitivity. The substitutional doping of $\mathrm{Sn}$ ions in $\mathrm{SnO}_{2}$ by Mo ions can construct oxygen vacancies, thus providing more active sites to adsorb alcohol gas ${ }^{17}$. Additionally, Lima et al. ${ }^{18}$ explored a $\mathrm{Ni}$ mesh coated with $\mathrm{Ni}(\mathrm{OH})_{2}$ nanoparticles to detect the concentration of alcohol by the change in the oxidation current. These studies represent the rapid development of alcohol detection technology and hint at the possibility of using such technology to detect food freshness. Recently, Gao et al. ${ }^{19}$ adopted keel-type $\mathrm{ZnO}$ fibers to fabricate a highly sensitive and selective alcohol detection. The unique grooves and meso- and macroporosities of the keel-type $\mathrm{ZnO}$ facilitated the fast diffusion and adsorption of alcohol. The fluorinated $\mathrm{SnO}_{2}$ films was used to fabricate a volatile organic compound sensor, which exhibited a ppm-level response to target gases with specificity. The fluorination promoted the persistent photoconductivity and accelerated the recovery process ${ }^{20}$. Zbořil et al. ${ }^{21}$ found that low concentration ofalcohol prevented the fluorescence quenching of highly fluorescent carbon nanoparticles during the liquid-to-solid phase transition, and made use of that to develop a fluorescence "turn- 
on" alcohol sensor to detect alcohol among other interferents with a low detection limit (100 ppm), which offered a great potential for diagnosis of early stage lung cancer. Shen et al. ${ }^{22}$ acidified $\mathrm{SnO}_{2}$ to fabricate the acidified $\mathrm{SnO}_{2} / \mathrm{rGO}$ aerogel with $\mathrm{rGO}$, which contributed to ethanol gas sensing under ultralow concentration owing to sufficient active sites and adsorption pores. This gas sensor exhibited an excellent response to alcohol. However, the device cost and real-time responsiveness should not be ignored. These above devices were usually fabricated by multi-treatment at high temperature and/or high pressure. The output signals of those were not visible to the naked eye and needed to be calculated through computer or converted by sensors, which increased the cost and response time. Considering those above mentioned reasons, we proposed a strategy based on light transmittance response to alcohol, which required matching refractive index (RI) between porous materials and the alcohol. When the pores were filled by alcohol, they immediately exhibit a light transparency that was visible to the naked eye. Meanwhile, the materials could also restore opaque with the vapor of alcohol. Thus, the alcohol detection based on light transmittance response could exhibit not only a real-time response but also reliable reusability. Because alcohol and acetic acid are the main products during the rotting of fruits and their refractive indices are 1.329 and 1.3716 respectively ${ }^{23-24}$, we considered preparing a porous polymer matrix with a matching RI, and then infiltrating it by alcohol and/or acetic acid to replace the air in the pores. The infiltrated membrane was full of a polymer matrix and alcohol/acetic acid, which could improve the amount of transmitted light, resulting 
in a real-time light transmission response. The RI of most polymers ranges from 1.4 to 1.6 due to the existence of aromatic groups, halogens (except fluorine), silicon, phosphorus, and organometallic moieties, while that of poly(vinylidene fluoride-cohexafluoropropylene) (PVDF-HFP) with rich fluorine groups was close to that of alcohol ${ }^{25}$. Due to its low RI, excellent hydrophobicity, and desired dimensional stability, PVDF-HFP was employed as the electrospinning matrix to achieve porous packaging ${ }^{26}$. Besides, the formulation also includes a natural polysaccharide sodium alginate (SA), whose RI was closer to that of alcohol/acetic acid compared with that of PVDF-HFP. Thus, the addition of SA to modify PVDF-HFP could further decrease light scattering. SA has been widely applied in biomedical, purification, and food packaging owing to its biocompatibility, biodegradability and ease of functionalization. However, the insufficient chain entanglements caused by high chain rigidity and strong electrostatic repulsion among SA polyanion chains could result in many difficulties in electrospinning. The surface tension difference between the aqueous phase of SA solution and the organic solvent phase of PVDF-HFP solution further increases the challenges for electrospinning ${ }^{27}$. Herein, we attempted to add a water-soluble component with excellent compatibility and electrospinnability to offer sufficient entanglement to the SA precursor and then selectively remove the auxiliary component. Besides, the original as-spun membrane was crosslinked by multivalent cations such as $\mathrm{Ca}^{2+}$ and became a stable three-dimensional network with water resistance posttreatment. 
In this work, we electrospun a core-shell structured SA@PVDF-HFP and applied it in intelligent packaging based on transmittance response to alcohol in order to monitor the freshness of fruits and vegetables. As time goes by, when the decay of grape intensifies with the effect of microorganisms, the decay product alcohol fills the fiber pores, which increases light transmittance, as shown in Figure 1. PVDF-HFP was selected as the shell material to provide effective water resistance and polyvinyl alcohol (PVA) was employed to improve the electrospinnability of the SA core. Then, the membrane was immersed in $\mathrm{CaCl}_{2}$ ethanol solution to improve the water stability and the PVA component was water-flushed to establish a tight substrate with less air-fiber interfacial area. Different SA concentrations were used, the interior structure and transparency of the fibers were investigated, and the mechanism was studied. Then, the as-spun membrane was used to cover a grape for several days to illustrate its potential for intelligent packaging. The result suggests that the as-prepared core-shell structured SA@PVDF-HFP nanofibrous membrane would be competent in multifarious relevant challenging settings and possess a broad application prospect in the field of food packaging. 


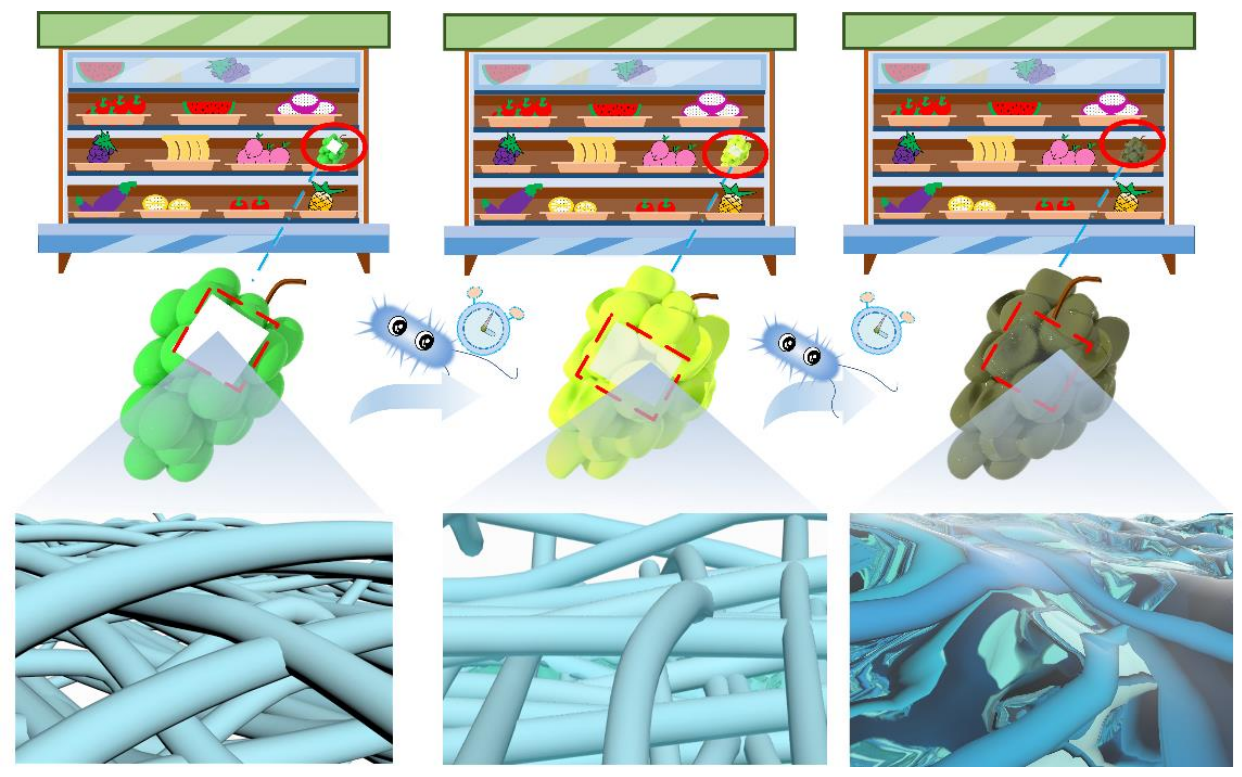

Figure 1. A naked-eye detector based on rapid transmittance response to alcohol using core-shell structured nanofibers. The transmittance change of the as-spun membrane is driven by the response to the concentration of rotten products, such as alcohol and acetic acid.

\section{Experimental Section}

\subsection{Materials}

PVDF-HFP (molecular mass 450000) was purchased from RHAWN. SA and PVA (molecular weight $\sim 67000$ ) were purchased from Aladdin. $\mathrm{CaCl}_{2}, \quad \mathrm{~N}, \mathrm{~N}$ dimethylformamide (DMF), ethanol absolute, sodium hydroxide $(\mathrm{NaOH})$ and $\mathrm{N}$-butanol were purchased from Tianjin Xinbaite Chemical Co., Ltd. Hydrochloric acid ( $\mathrm{HCl})$, sodium chloride $(\mathrm{NaCl})$ and acetone were purchased from Tianjin Xintong Chemical Co., Ltd. Sunflower seed oil was purchased from Shanghai Fulinmen Food Co., Ltd. 
Deionized water was produced using a laboratory water purification system (SmartQ15 supplied by Hitect Instruments Co., Ltd.

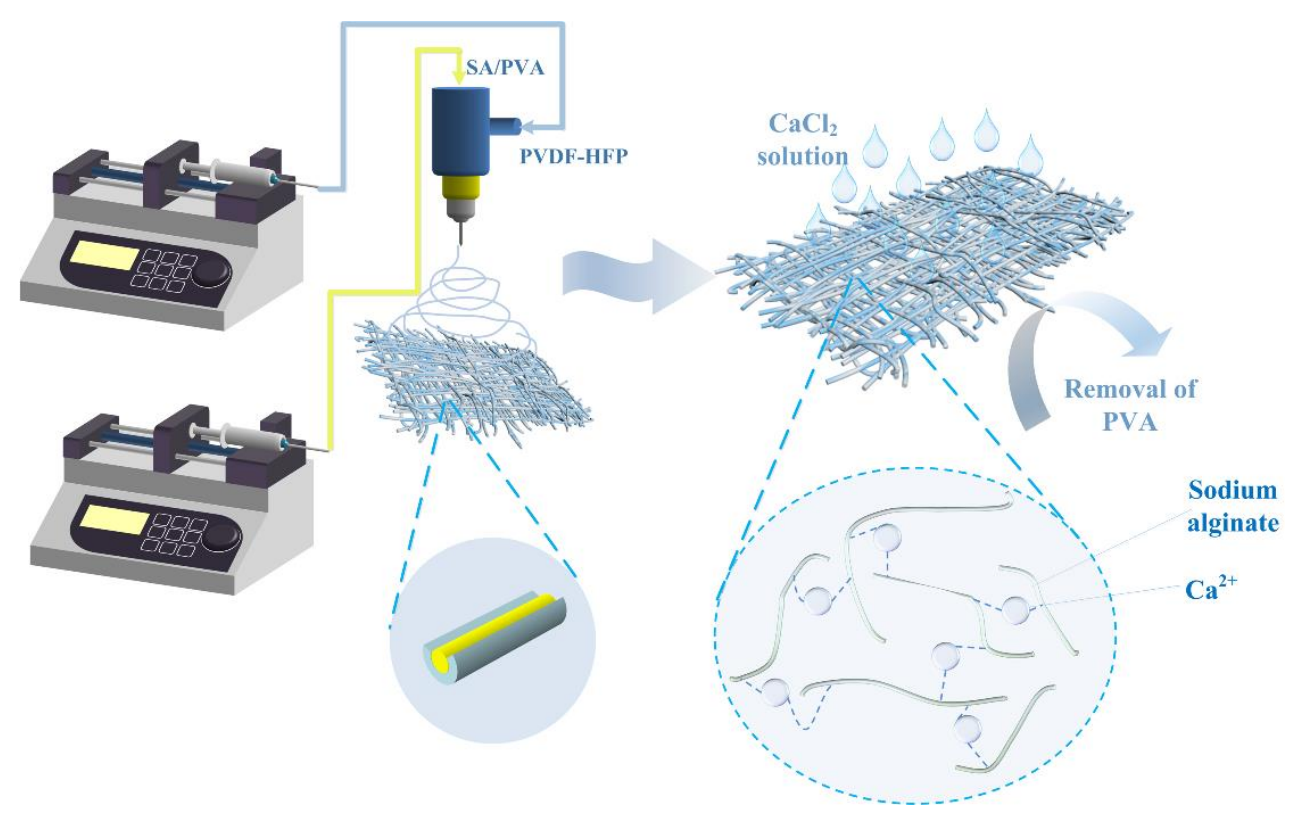

Figure 2. Preparation processes of SA/PVA@PVDF-HFP nanofibrous membrane.

\subsection{Fabrication of Intelligent Packaging}

The preparation process for intelligent packaging was shown in Figure 2. The electrospinning solution contained $15 \mathrm{wt} \%$ PVDF-HFP in DMF and acetone (7/3, w/w), and a certain concentration $(0,0.5,1$, or $2 \%, \mathrm{w} / \mathrm{w})$ of SA was dissolved in a $10 \mathrm{wt} \%$ PVA aqueous solution. The two spinning solutions were vigorously stirred respectively at room temperature for $24 \mathrm{~h}$. A coaxial nozzle was utilized to fabricate electrospun SA/PVA@PVDF-HFP core-shell fibers, where PVDF-HFP was selected as the shell material, and SA/PVA was employed as the core material. They were placed in $5 \mathrm{~mL}$ syringes respectively and controlled by programmed syringe pumps (TYD01-01, 
Baoding Lead Fluid Technology Co., Ltd., China) with different feeding rates. The feed rate of PVDF-HFP was fixed at $0.6 \mathrm{~mL} / \mathrm{h}$, and that of SA/PVA was at $0.1 \mathrm{~mL} / \mathrm{h}$. The aluminum foil with the dimension of $5 \times 5 \mathrm{~cm}^{2}$ was adopted as collector. The collecting time of all as-spun membranes was fixed at $3 \mathrm{~h}$. The environmental humidity was maintained around $18 \%-30 \%$ and the temperature was $25 \pm 2^{\circ} \mathrm{C}$. A power supply (DE100, Dalian Dingtong Technology Co., Ltd., China) provided $16 \mathrm{kV}$ high voltage with a nozzle-to-collector distance of $15 \mathrm{~cm}$.

To ensure the water resistance of SA during further water-flushing of the PVA component, the membrane was treated in a $2 \mathrm{wt} \% \mathrm{CaCl}_{2}$ ethanol solution for $24 \mathrm{~h}$ until the $\mathrm{Ca}^{2+}$ was crosslinked with SA to form hydrogel. Then the fibrous membrane was repeatedly rinsed with deionized water several times to remove the PVA. The obtained fibrous membranes were dried at $40{ }^{\circ} \mathrm{C}$.

\subsection{Characterization}

\subsubsection{Solution Properties}

The solution viscosity was determined by a rotary viscometer (NDJ-1, Shanghai Yutong Instrument Factory, China); the conductivity was examined by a conductivity meter (DDS-307, INESA Scientific Instrument Co., Ltd., China), and the surface tension was determined using a surface tension meter (JYW-200B, Youte Electronics Co., Ltd., China). All average value of three determinations was obtained and reported as mean \pm standard deviation (SD). 


\subsubsection{Fourier Transform Infrared Spectroscopy}

A Fourier-transform infrared spectroscopy spectrometer (TENSOR 27, Brooke, Germany) was used to analyze the chemical structure over a range of $4000-600 \mathrm{~cm}^{-1}$.

\subsubsection{Scanning Electron Microscopy (SEM)}

The surface morphology of the fibrous membranes was observed by a field-emission scanning electron microscope (SEM, JSM-6700F, JEOL, Japan). The diameters were measured using Image $\mathbf{J}$ software and at least 100 different fiber segments were randomly selected from each image.

\subsubsection{Transmission Electron Microscopy}

A transmission electron microscope (JEM-2100F, JEOL, Japan) was used to observe the core-shell structure of the as-spun nanofibers at $200 \mathrm{kV}$ electron beam accelerating voltage. The samples to measure by TEM were electrospun directly on copper meshes.

\subsubsection{X-ray Photoelectron Spectroscopy (XPS)}

The chemical composition of the SA/PVA@PVDF-HFP membrane surfaces was examined by X-ray photoelectron spectroscopy (ESCLab220i-XL, VG, USA) with C 1 s, $\mathrm{O}_{1 \mathrm{~s}}$, and $\mathrm{F}_{1 \mathrm{~s}} \mathrm{X}$-ray sources.

\subsubsection{Contact Angle Test}


A contact angle (CA) measuring instrument (DSA100, Krüss, Germany) was used to determine the surface wettability of the SA/PVA@PVDF-HFP and SA@PVDF-HFP fibrous membranes. Approximately a $2 \mu \mathrm{L}$ liquid droplet was placed on the surface of the as-spun membranes and analyzed by the computer software. The average CA values were from three measurements on different positions per sample.

\subsubsection{UV-Vis Spectrophotometer}

The light transmittance of the membranes was observed on a T6 New Century UV-vis Spectrophotometer (Persee, Beijing, China).

\subsubsection{X-ray Diffraction}

The crystal structures of the SA/PVA@PVDF-HFP fibers were characterized by wideangle X-ray scattering (D/Max 2500pc, Rigaku, Japan). A scanning angle range of $5-90^{\circ}$ and a scanning rate of $4 \%$ min were used.

\subsubsection{Porosity Measurements}

A fibrous membrane (weight $\omega_{0}$ ) was put into $\mathrm{N}$-butanol solution, which was put into a vacuum oven, standing for $6 \mathrm{~h}$, before it was taken out and weighed. At a certain time point, the weight was recorded as $\omega_{t}$.

$$
P=\frac{\omega_{t}-\omega_{0}}{\rho V} \times 100 \%(1)
$$


Where $P$ is the porosity of the membrane, $\omega_{0}$ is the weight of the membrane before immersion, $\omega_{t}$ is the weight of the membrane after immersion for a certain time, $\rho$ is the density of absolute ethanol and $N$-butanol, and $V$ is the volume of the fibrous membrane.

\subsubsection{Mechanical Properties Measurements}

The mechanical properties of fibrous membranes was measured on the tensile testing machine (Z005, Zwick/Roell, GmbH Ulm, Germany) using a tensile rate of $30 \mathrm{~mm} / \mathrm{min}$ at ambient temperature. The gauge length of the sample was constant at $30 \mathrm{~nm}$, and the load cell was fixed at $2500 \mathrm{~N}$.

\subsubsection{Performance of the Intelligent Packaging}

2\%SA@PVDF-HFP covered on fresh fruit (Jufeng grapes obtained from the Campus grocery) was used in this experiment. It was placed in an environment of $32.8^{\circ} \mathrm{C}$ and $59 \%$ relative humidity. Its optical transparency was measured every 2 days with a light transmittance meter (LH-220, Lianhuicheng, China). For the reusability test, the light transmittance of fibrous membranes was measured immediately after being wetted with alcohol. After drying at room temperature, the light transmittance was tested again and repeated 10 times. In order to test the performance of membranes in various situations, the transmittance response to $\mathrm{HCl}$ solution $(\mathrm{pH}=3), \mathrm{NaOH}$ solution $(\mathrm{pH}=12), 0.9 \%$ $\mathrm{NaCl}$ solution and sunflower seed oil was measured.

\section{Results and Discussion}




\subsection{Morphology of Electrospinning SA@PVDF-HFP}

Figure 3 presented typical SEM images of the SA/PVA@PVDF-HFP nanofibers with different SA concentrations. It is noteworthy that in 0.5\%SA/PVA@PVDF-HFP and 2\%SA/PVA@PVDF-HFP, nanofibers were organized to form a randomly oriented beads structure on the micro-scale only, which could be caused by the precursor properties such as viscosity, surface tension, and conductivity. Among those, viscosity played a role in maintaining jet stability. The viscosity was too large $(>2000 \mathrm{mPa} \cdot \mathrm{s})$ to maintain the stability of the jet ${ }^{28}$. Without the SA component, uniform and cylindrical fibers were obtained. With the addition of SA, the insufficient entanglement of SA molecules led to aggregation in the core to some extent, which resulted in a bead-fiber structure. As the SA concentration increased to $1 \%$, the viscosity was enough to maintain a stable jet and as a result, continuous fibers were generated. When the SA concentration was $2 \%$, the viscosity was up to $9800 \mathrm{mPa} \cdot \mathrm{s}$ well beyond the viscosity range for maintaining a stable jet (as shown in Table 1), which was difficult to be sufficiently stretched by the traction forces caused by the conductivity. To sum up, only when the concentration of SA was $0 \%$ or $1 \%$ can the charge repulsion and surface tension reach an unsteady equilibrium and uniform fibers be produced.

The diameters of nanofibers were also determined by the properties of the precursors including viscosity, surface tension, and conductivity. When the viscosity was more than $2000 \mathrm{mPa} \cdot \mathrm{s}$, the stretching forces accounting for the conductivity failed to overcome the surface tension and thus the surface tension of the electrospun precursors 
dominated; otherwise, the conductivity would dominate ${ }^{29}$. At the same time, a negative correlation between conductivity and fiber diameter was evident and the surface tension had a highly positive correlation with fiber diameter ${ }^{30}$. With increasing SA concentration from $0 \%$ to $1 \%$, the diameter of the as-spun fiber decreased from 336.1 $\mathrm{nm}$ to $187.4 \mathrm{~nm}$ due to the conductivity increasing for the low viscosity precursor. In contrast, when the SA concentration was $2 \%$, the viscosity increased significantly to $9800 \mathrm{mPa} \cdot \mathrm{s}$. The diameter decreased to $206.8 \mathrm{~nm}$ owing to a more dominant role of surface tension. This phenomenon was explained by the competition between surface tension and conductivity, and which one of them was the dominant factor depends on the viscosity of the as-spun precursor.

The morphology of as-spun fibrous membranes crosslinked by $\mathrm{CaCl}_{2}$ was shown in Figure $\mathrm{S} 1$. After crosslinking by a $\mathrm{CaCl}_{2}$ ethanol solution, $\mathrm{SA}$ formed ionic crosslinks with $\mathrm{Ca}^{2+}$, while PVA swelled in alcohol and the volume increased. For 0\%SA/PVA@PVDF-HFP without a core-shell structure, PVA was swelled and partially dissolved in alcohol after a long time, and the fibrous morphology deteriorated and the surface collapsed after crosslinking. After water-flushing, the complete fibrous morphology remained as shown in Figure S2. Regarding this phenomenon, a stable SA hydrogel was formed by crosslinking with $\mathrm{Ca}^{2+}$, leading to dramatically increased fiber diameter and membrane thickness. Even 1\%SA/PVA@PVDF-HFP was cracked by the swelling PVA and SA hydrogel while 2\%SA/PVA@PVDF-HFP exhibited a smooth surface as shown in Figure $5^{31}$. The diameter and thickness of the as-spun membranes 
were slightly decreased, and a slight collapse was observed owing to the removal of PVA. Due to the absence of SA, there was no hydrogel in 0\%SA/PVA@PVDF-HFP, and the diameter decreased after the PVA was removed.
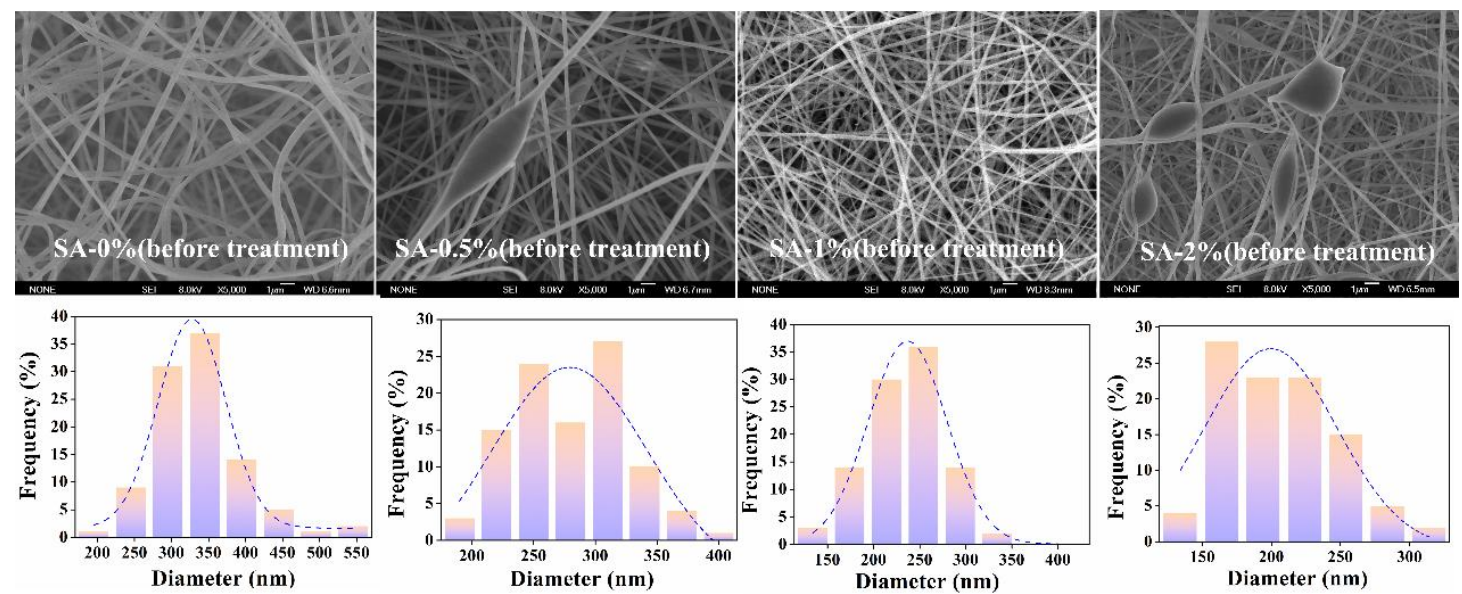

Figure 3. SEM images and diameter distributions of the as-spun SA/PVA@PVDFHFP nanofibers with different SA concentrations.

Table 1 Solution Properties of the Electrospinning Precursors and the Average Fiber Diameter (AFD) of the As-Spun Fibers.

\begin{tabular}{lcccccc}
\hline Sample & $\begin{array}{l}\text { Conductivity } \\
\left(\mu \mathrm{s} \cdot \mathrm{cm}^{-1}\right)\end{array}$ & $\begin{array}{l}\text { Viscosity } \\
(\mathrm{mPa} \cdot \mathrm{s})\end{array}$ & $\begin{array}{l}\text { Surface } \\
\text { tension } \\
\left(\mathrm{mN} \cdot \mathrm{m}^{-1}\right)\end{array}$ & $\begin{array}{l}\text { AFD } \\
\text { before } \\
\text { treatment } \\
(\mathrm{nm})\end{array}$ & $\begin{array}{l}\text { AFD after } \\
\text { treatment } \\
(\mathrm{nm})\end{array}$ & $\begin{array}{l}\text { AFD after } \\
\text { crosslinking } \\
(\mathrm{nm})\end{array}$ \\
\hline PVDF-HFP & $5.8 \pm 0.5$ & $375 \pm 10.8$ & $6.4 \pm 0.4$ & - & - & - \\
PVA & $37.6 \pm 1.5$ & $52 \pm 2.8$ & $1.2 \pm 0.3$ & 336.1 & 233.3 & - \\
$\begin{array}{l}\text { 0.5\%SA/PV } \\
\text { A }\end{array}$ & $79.0 \pm 3.5$ & $185 \pm 10.8$ & $10.7 \pm 0.6$ & 280.1 & 301.6 & - \\
$1 \%$ SA/PVA & $148.3 \pm 2.9$ & $1450 \pm 122.5$ & $33.0 \pm 2.6$ & 187.4 & 277.0 & 499.6 \\
$2 \%$ SA/PVA & $261.0 \pm 10.9$ & $9800 \pm 282.8$ & $40.9 \pm 1.5$ & 206.8 & 267.9 & 326.2 \\
\hline
\end{tabular}




\subsection{Composition of Electrospinning SA@PVDF-HFP}

ATR-FTIR was used to characterize the chemical structure of the surface of the nanofiber samples and the spectra were obtained over a range of $600-4000 \mathrm{~cm}^{-1}$. As shown in Figure 4 (a), the peaks at 1400, 1278, 1178, 1070, 868, 820, and $610 \mathrm{~cm}^{-1}$ can be assigned to typical characteristic peaks of PVDF-HFP. The peaks at 1400, 1178 and $1070 \mathrm{~cm}^{-1}$ were attributed to the stretching vibrations of $\mathrm{CH}_{2}$ and $\mathrm{CF}_{2}$ and the out-ofplane deformation of $\mathrm{CF}_{3}{ }^{32}$. The bands at around 1278 and $868 \mathrm{~cm}^{-1}$ represent the electroactive $\beta$ phase of the PVDF-HFP nanofibers and the peak at $610 \mathrm{~cm}^{-1}$ is ascribable to the $\alpha$ phase ${ }^{33-34}$.

With the addition of a range of concentrations of PVA/SA, the same characteristic peaks at 1400, 1278, 868, 820, and $610 \mathrm{~cm}^{-1}$ arising from PVDF-HFP could also be observed. In comparison, for 0\% and 0.5\%SA/PVA@PVDF-HFP, additional peaks at 2923 and $1076 \mathrm{~cm}^{-1}$ assigned to the $\mathrm{C}-\mathrm{H}$ and $\mathrm{C}-\mathrm{O}$ moieties of PVA were observed. Besides, when the content of SA were $0 \%$ and $0.5 \%$, the strong peak at $1076 \mathrm{~cm}^{-1}$ assigned to the skeletal vibration involving the bridge $\mathrm{C}-\mathrm{O}$ stretching of PVA covered the peak at $1070 \mathrm{~cm}^{-1}$ for $\mathrm{CF}_{3}{ }^{35}$. Moreover, there was a broad peak spanning from 3000 to $3500 \mathrm{~cm}^{-1}$, which can be attributed to the $\mathrm{O}-\mathrm{H}$ vibration of PVA and/or SA, while this peak was absent in the composite fibers when the SA concentration was $1 \%$ or higher. When the content of SA raised to $1 \%$ and $2 \%$, the characteristic peaks of PVA and SA disappeared, whose spectra curves were the same as those for pure PVDF-HFP. Therefore, it could be inferred that for the fibers with lower SA concentrations $(0 \%$ and 
0.5\%), PVDF-HFP did not completely wrap SA/PVA and part of the core component was exposed to the outside of the shell.

Figure 4 (c) showed the X-ray diffraction (XRD) patterns of pure PVDF-HFP, 0\%SA/PVA@PVDF-HFP, 0.5\%SA/PVA@PVDF-HFP, 1\%SA/PVA@PVDF-HFP, and 2\%SA/PVA@PVDF-HFP. The pure PVDF-HFP membrane exhibited two diffraction peaks at $18.4^{\circ}\left(\begin{array}{lll}1 & 0 & 0\end{array}\right)$ and $20.2^{\circ}\left(\begin{array}{lll}0 & 2 & 0\end{array}\right)$ related to the formation of the semicrystalline phase ( $\beta$ phase $)^{36}$. Electrospun PVA was amorphous and the addition of highly crystalline SA did not lead to crystallization of PVA ${ }^{37}$. With the addition of SA, 0.5\%SA/PVA@PVDF-HFP and 1\%SA/PVA@PVDF-HFP presented multiple sharp crystalline peaks at $21.6^{\circ}, 24^{\circ}$, and $26.8^{\circ}$ attributed to the $\alpha$ phase of SA ${ }^{38}$, and 2\%SA/PVA@PVDF-HFP presented the same crystalline structure as pure PVDF-HFP. This phenomenon was associated with insufficient traction and stretching in the electrospinning process due to the relatively higher viscosity and surface tension of $2 \% \mathrm{SA} / \mathrm{PVA}$.
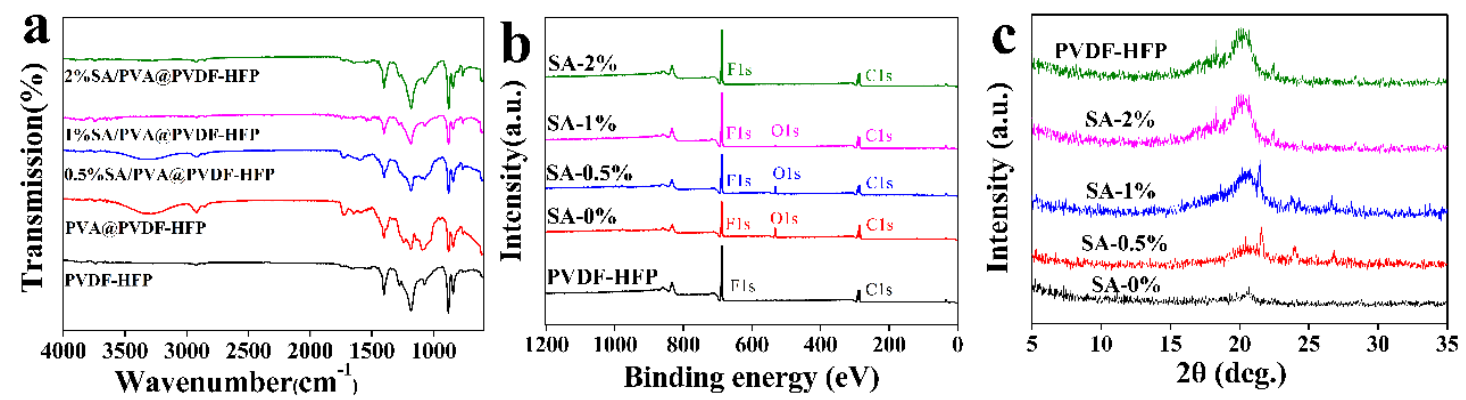

Figure 4. (a) FTIR spectra for SA/PVA@PVDF-HFP samples with different SA concentrations. (b) XPS spectra for SA/PVA@PVDF-HFP samples with different SA 
concentrations and pure PVDF-HFP. (c) XRD curves for SA/PVA@PVDF-HFP samples with different SA concentrations and pure PVDF-HFP.

\subsection{Interior Structure of Electrospinning SA@PVDF-HFP}

Figure 7 shows TEM images of the interior structure of the SA/PVA@PVDF-HFP asspun nanofibers. As shown in Figure 7 (a), without the addition of the SA component, there was no core-shell structure but a non-concentric structure. Different from that, 0.5\%SA/PVA@PVDF-HFP displayed an evolution from the non-concentric structure to a core-shell structure and SA/PVA was incomplete wrapped by PVDF-HFP as shown in Figure 7 (b), which could be attributed to the higher viscosity and stronger surface tension than that of $0 \% \mathrm{SA} / \mathrm{PVA}$. A typical core-shell structure was shown in Figure 7 (c-d), and the diameter ratio (inner diameter/outer diameter) of 1\%SA/PVA@PVDFHFP and 2\%SA/PVA@PVDF-HFP was 77.1\% and 66.7\%, respectively, which is mainly attributed to the charge attractive forces of the Taylor cone.

The elements of the nanofiber surface ranging from 3 to $10 \mathrm{~nm}$ were detected by XPS and the intensity of the diffraction peaks was attributed to the contents of elements. As shown in Figure 4 (b), the intensity of $\mathrm{F}_{1 \mathrm{~s}}$ increased and the intensity of $\mathrm{O}_{1 \mathrm{~s}}$ decreased with a higher content $(0,0.5,1$, and $2 \mathrm{wt} \%)$ of SA. As the F element existed only in PVDF-HFP, the increase in the $\mathrm{F}_{1 \mathrm{~s}}$ intensity was ascribed to the better wrapping to form a core-shell structure, which was consistent with TEM results. Nonetheless, the intensity of $\mathrm{F}_{1 \mathrm{~s}}$ in $2 \% \mathrm{SA} / \mathrm{PVA} @ \mathrm{PVDF}-\mathrm{HFP}$ was still weaker than that for pure PVDF- 
HFP, which can be attributed to the formation of a very small amount of incomplete core-shell structure in the former.

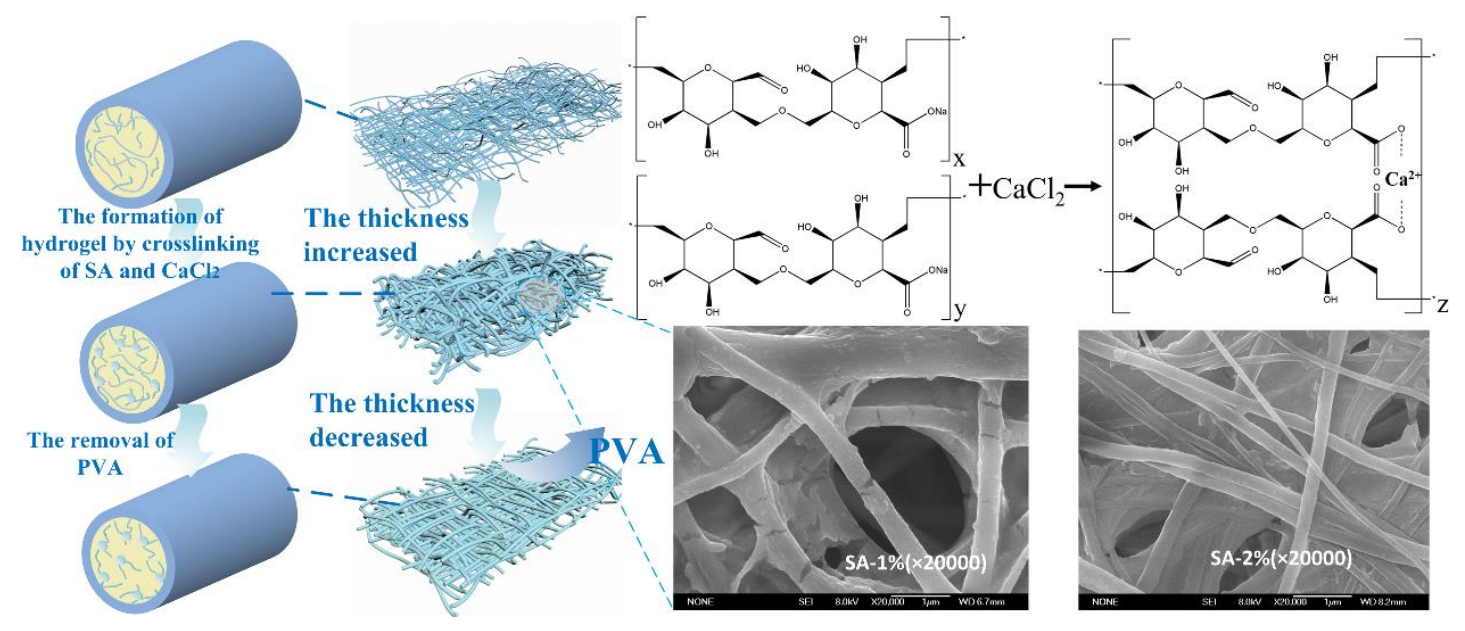

Figure 5. Process and mechanism of the crosslinking of SA with $\mathrm{Ca}^{2+}$, and the removal of PVA (On the top right is the reaction equation describing the crosslinking of SA with $\mathrm{Ca}^{2+}$ and on the bottom right are SEM images at high magnification of $1 \%$ and 2\%SA/PVA@PVDF-HFP after crosslinking).

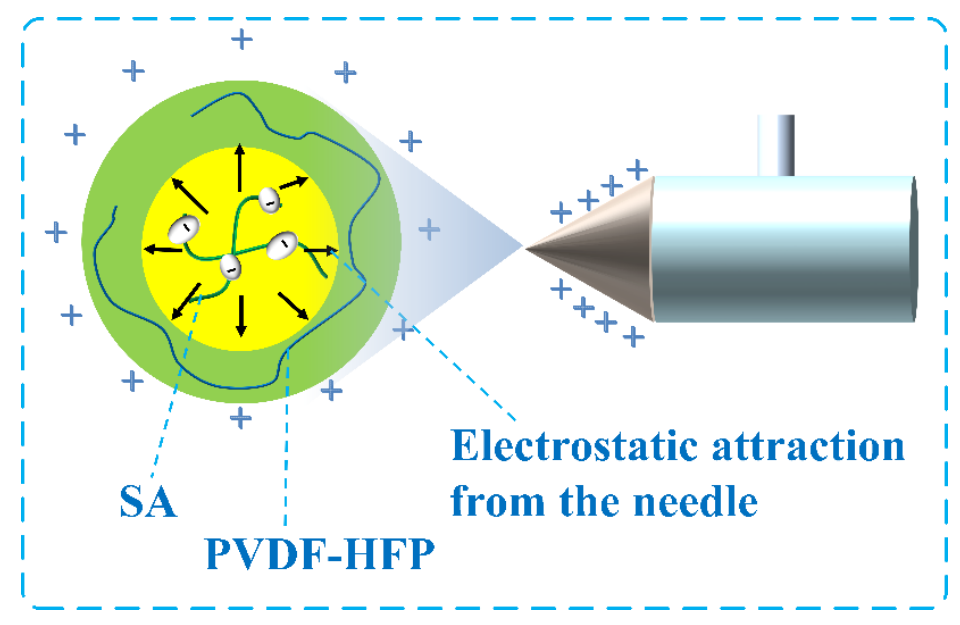

Figure 6. During electrospinning, SA was attracted by the surface of the Taylor cone, as a positive-polarity electric source. 
The mechanism regarding the evolution of the fiber interior structure was illustrated in Figure 6 and Figure 7. There was strong conductivity of the SA aqueous solution. During electrospinning, the applied electric field made the Taylor cone become the positive polarity of the electric field source and the anionic polyelectrolyte SA had a higher tendency to exhibit electroresponsive behavior and was directed towards the electrospun jet surfaces ${ }^{31}$. Without the introduction of SA, the PVA aqueous solution with high flowability was incompatible with the PVDF-HFP precursor, leading to a non-concentric interior structure via coaxial electrospinning, as shown in Figure 7 (a). With the addition of SA, the viscosity and surface tension of $0.5 \% \mathrm{SA} / \mathrm{PVA}$ increased. The entanglement of molecules decreased the SA/PVA asymmetric mobility, but it was still hard to overcome the charge attractive forces by the surface of the Taylor cone to form a complete core-shell structure. A complete core-shell structure was formed in 1\%SA/PVA@PVDF-HFP and 2\%SA/PVA@PVDF-HFP. However, the viscosity and surface tension of $2 \% \mathrm{SA} / \mathrm{PVA}$ was much higher than that of $1 \% \mathrm{SA} / \mathrm{PVA}$, whose core material was more difficult to be pulled by charge attractions when being ejected from the nozzle, resulting in a smaller diameter ratio. 


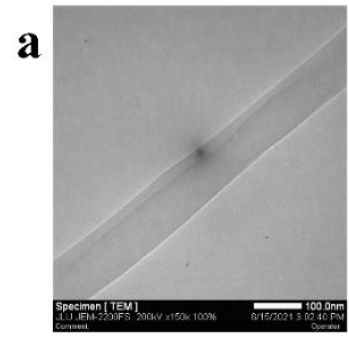

C

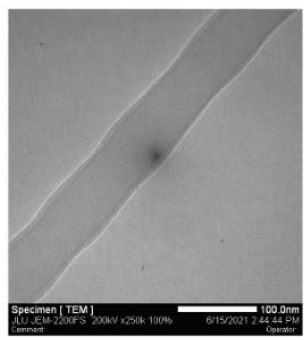

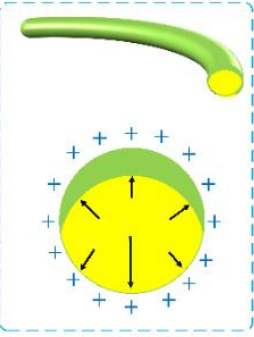

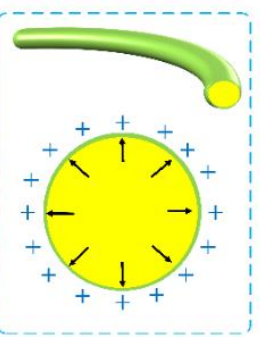

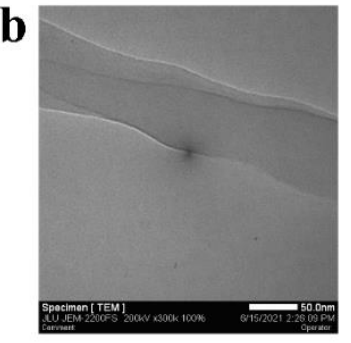

d

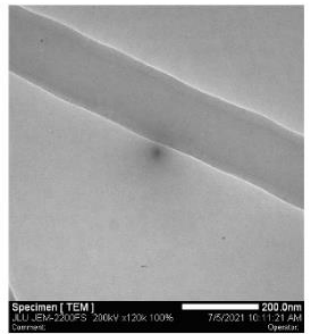

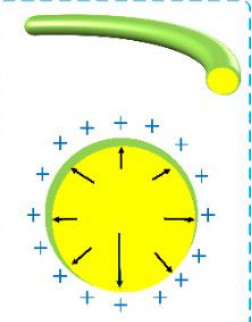

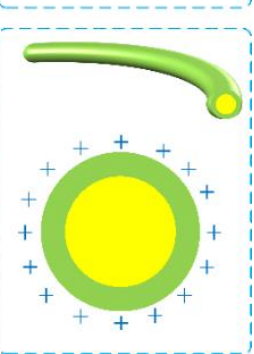

Figure 7. (a-d) TEM images and the interior structure of the SA/PVA@PVDF-HFP asspun nanofibers with $0 \%, 0.5 \%, 1 \%$ and $2 \%$ SA contents.
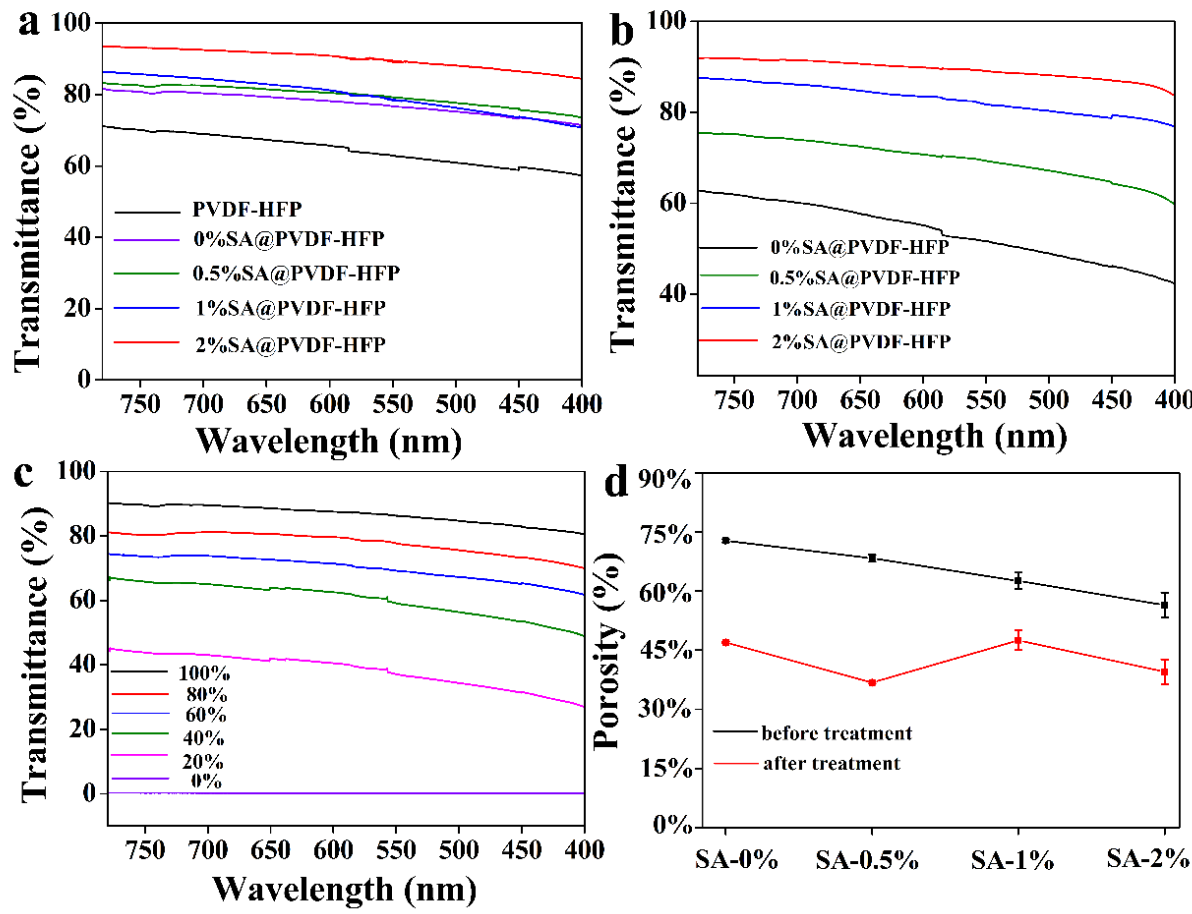

Figure 8. (a) UV-vis spectra for pure PVDF-HFP and SA@PVDF-HFP samples with different SA contents wetted by alcohol. (b) UV-vis spectra for SA@PVDF-HFP samples with different SA contents wetted by acetic acid. (c) UV-vis spectra for 
2\%SA@PVDF-HFP infiltrated by different concentrations of ethanol solution. (d) Porosity of SA/PVA@PVDF-HFP and SA@PVDF-HFP after treatment with different SA concentrations.

\subsection{Transparency Analysis}

The UV-vis spectra of the membranes are shown in Figure 8 (a-c). All membranes were opaque in the visible range. After alcohol infiltration, the as-spun membranes became optically transparent with different transmittance as shown in Figure 8 (a). The transmittance was enhanced with an increase in the SA concentration, and when the SA concentration reached $2 \%$, the maximum content of electrospinning, the light transmittance reached the maximum. The phenomenon was attributed to the combined effects of the diameter and concentration of SA.

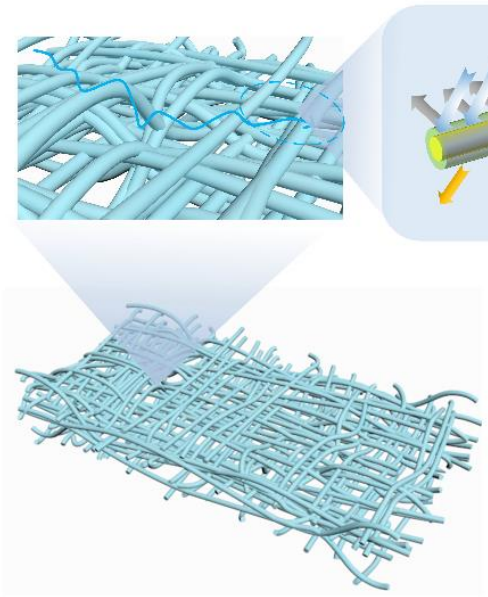

The as-spun fibrous membranc before immersed by alcohol
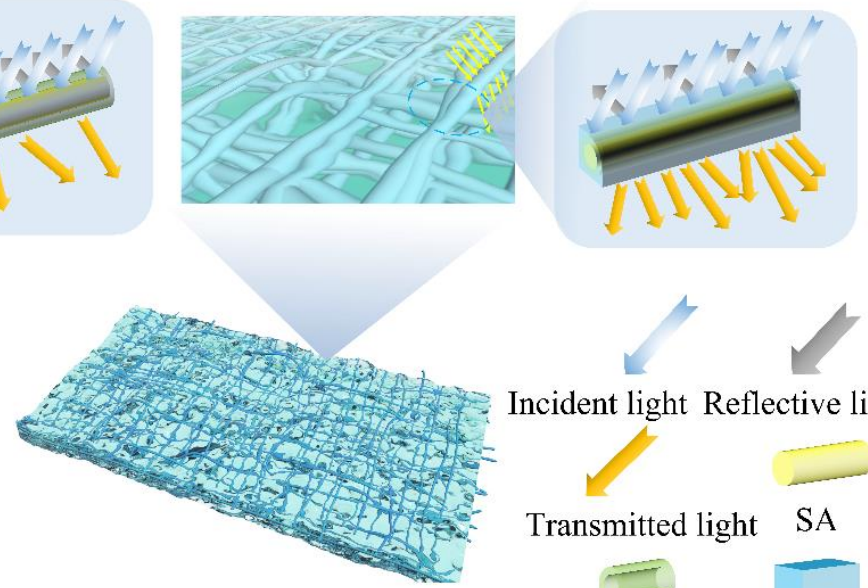

The as-spun fibrous membrane after immersed by alcohol
Incident light Reflective light

Transmitted light SA

PVDF-HFP Alcohol 
Figure 9. Schematic diagram of the influence of RI and as-spun fiber diameter on light propagation before and after being wetted by alcohol and acetic acid (the number of arrows represents light intensity).

The decrease in transmittance was associated with not only the reflection/refraction of light at the interface between fiber and air but also the absorption within fibers. There was a large air-fiber interfacial area as shown in Figure 9, where the light was supposed to scatter severely, resulting in opaqueness. However, the membranes became optically transparent after alcohol treatment, which could be explained by the following. First and foremost, RI discrepancy between the nanofiber and air was highly related to the amount of light loss, and the relationship is deduced by the following formula.

$$
\Gamma=\left[\frac{\left(n_{1}-n_{2}\right)}{\left(n_{1}+n_{2}\right)}\right]^{2}(2)
$$

Where $\Gamma$ is the reflection coefficient, $n_{1}$ and $n_{2}$ are the RI values of the air and fiber at the interface respectively. The more the discrepancy in RI between fibers and the surrounding, the higher the $\Gamma$ is. The RI of alcohol was 1.329 , close to those of PVDFHFP (1.3380) and SA (1.3343) and the RI of air was 1 at $20^{\circ} \mathrm{C}, 760 \mathrm{mmHg}$. As shown in Figure 9, after the pores of membranes were filled with alcohol, the light reflection at the fiber-alcohol interface was much lower than that at the fiber-air interface, resulting in higher light transmittance of the membranes after alcohol infiltration. Owing to the closer RI of SA to that of alcohol compared with PVDF-HFP, the addition of SA effectively increased the light transmittance of composite as-spun membranes as 
shown in Figure 8 (a). As the content of SA increased, the RI discrepancy between fibers and alcohol decreased, resulting in high transparency. Additionally, the diameter also had a dominant effect, affecting the propagation of light. Visible light, as an electromagnetic wave could pass fibers of $<400 \mathrm{~nm}$ in diameter without reflection and refraction at the fiber-surrounding interface. Hence, the reason for the high transparency is that the diameter of all fibers after treatment was lower than $400 \mathrm{~nm}$, as shown in Table 1. Additionally, as shown in Figure 8 (d), the amount of the fiber-air interface decreased due to a slight collapse on the fiber surface, which led to reduced multi-reflection and improved light transmittance.

It is worth noting that the RI of acetic acid, another product of fruit decay is 1.3716 , also close to those of PVDF-HFP and SA. As a result, after treatment by acetic acid, the membranes exhibited optically transparent more than $60 \%$ as shown in Figure 8 (b). The transparency increased with a higher SA concentration. As the concentration of alcohol increased, the transparency was improved gradually as shown in Figure 8 (c).

\subsection{Wetting Properties of the Fibrous Membranes}

The water contact angle (WCA) is an effective index to indicate the hydrophobicity of the as-spun membrane surface. Owing to the hydrophobic PVDF-HFP shell layer, all the membranes were hydrophobic with WCA above $120^{\circ}$ as shown in Figure 10 (a). After treatment, the WCA of 0\%,0.5\%, 1\%SA@PVDF-HFP was slightly decreased, which was related to the destruction of the hydrophobic shell during treatment. And the 
WCA of 2\%SA@PVDF-HFP was increased attributed to the complete PVDF-HFP shell layer.

Alcohol and acetic acid were used to further analyze the wettability of the as-spun membranes. As shown in Figure 10 (b), the CA of alcohol and acetic acid were lower than $40^{\circ}$. The excellent wettability improved the transmittance response of the fibrous membranes to the alcohol and acetic acid.

\subsection{Application of the Fibrous Membranes in Intelligent Packaging}

The application of intelligent packaging for visual freshness detection is shown in Figure S3. The opaque SA@PVDF-HFP gradually became optically transparent, and the high transparency could reach 59.2\% as shown in Figure 10 (e), revealing the rotten part of the grape under the detector membrane. The high transmittance was obtained by the deterioration product, such as alcohol and acetic acid, which easily wetted the asspun membranes.
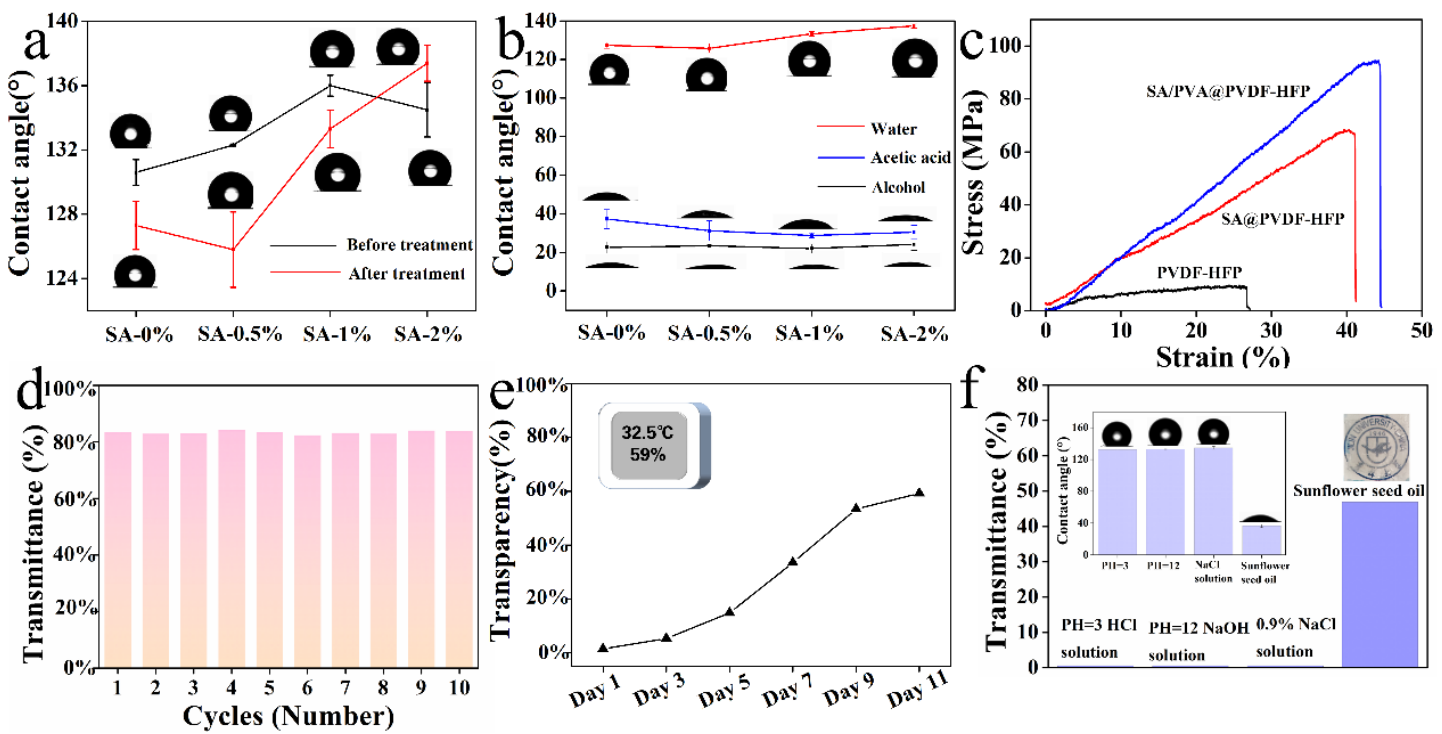
Figure 10. (a) WCA of SA@PVDF-HFP before and after treatment. (b)CA results of SA@PVDF-HFP with water, acetic acid, and alcohol. (c) Stress-strain curves of pure PVDF-HFP, SA/PVA@PVDF-HFP and SA@PVDF-HFP nanofibers. (d) Light transmittance response to alcohol changes with cycles. (e) Light transmittance during the 11 days. (f) The optical transmittance (at $550 \mathrm{~nm}$ ) and CA of the 2\%SA@PVDFHFP treated by different solutions.

As time went by, the alcohol and acetic acid concentration increased, which gradually infiltrated the fibrous membrane and filled the pores of the membrane. The result of the durability test was shown in Figure 10 (d). After ten times of alcohol infiltration and drying, the transparency of the fibrous membrane could still reach more than $80 \%$, and transparency was stable in a range from $82.9 \%$ to $84 \%$. Therefore, it can be concluded that the fibrous membrane had reliable sensitivity to alcohol and acetic acid for food freshness detection.

In order to better measure the performance of intelligent packaging in various situations , the transparency response to different solutions concluding $\mathrm{HCl}$ solution $(\mathrm{pH}=3)$, $\mathrm{NaOH}$ solution $(\mathrm{pH}=12), 0.9 \% \mathrm{NaCl}$ solution and sunflower seed oil was discussed, which was shown in Figure 10 (f). On account of the excellent hydrophobicity exceeding $130^{\circ}$ of the composite membranes in Figure. 10 (f) inset, it exhibited low light transmittance response to $\mathrm{HCl}$ solution $(\mathrm{pH}=3), \mathrm{NaOH}$ solution $(\mathrm{pH}=12)$ and $0.9 \%$ $\mathrm{NaCl}$ solution. Moreover, although the as-spun membranes were well infiltrated by the sunflower seed oil, the RI of sunflower seed oil from 1.461 to 1.468 was mismatched 
to that of the as-spun membrane, which resulted in a much lower light transmittance around $46.9 \%$.

The stress-strain curves of pure PVDF,2\%SA/PVA@PVDF-HFP and 2\%SA@PVDFHFP were shown in Figure 10 (c). The maximum tensile stress $\left(\sigma_{\max }\right)$, elongation at break $(\varepsilon b)$ and Young's modulus (E) were calculated from the stress-strain curves and summarized in Table S1. With the addition of PVA, the $\sigma_{\max }$ of 2\%SA/PVA@PVDFHFP increased from 11.24 MPa to $80.39 \mathrm{MPa}$, and E increased from 0.24 to $2.25 \mathrm{MPa}$. At same time, the $\varepsilon_{b}$ increased to $36.64 \%$. After crosslink and water-flushing, the $\sigma_{\max }$ slightly decreased to $56.69 \mathrm{MPa}$ and the E decreased to $1.31 \mathrm{MPa}$ assigned to the removal of PVA. However, compared with pure PVDF-HFP, the mechanical properties of that was effectively increased, due to the formation of SA hydrogel.

Importantly, the output signal of earlier reported alcohol detection needed to be calculated through computer or converted by sensors, which increased response time toward alcohol. Compared with those, the intelligent packaging was based on light transmittance response, which exhibited reusability for 10 times at least, and did not take time to convert the output signal (as shown in Table 2). Our work has potential in intelligent packaging to detect alcohol.

Table 2. Figure of Merits of intelligent packaging for detecting alcohol.

\begin{tabular}{cccc}
\hline target & response time (s) & response type & references \\
\hline methyl alcohol & $5 \mathrm{~s}$ & electrical response & 39
\end{tabular}




\begin{tabular}{lccc} 
alcohol & $20 \mathrm{~s}-30 \mathrm{~s}$ & chemical response & 40 \\
alcohol & $12.1 \mathrm{~s}$ & chemoresistive response & 19 \\
alcohol & $10 \mathrm{~s}$ & electrical response & 41 \\
alcohol & $2.5 \mathrm{~min}$ & electrical response & 42 \\
alcohol & $9.5 \mathrm{~min}$ & colorimetric response & 43 \\
alcohol & $9 \mathrm{~s}$ & electrical response & 44 \\
alcohol & real-time & transmittance response & This study \\
\hline
\end{tabular}

4 Conclusion

An intelligent packaging to real-time detection of food freshness can enhance food safety. In this work, we fabricated a core-shell structured SA@PVDF-HFP material highly responsive to alcohol by electrospinning and crosslinking with $\mathrm{CaCl}_{2}$ and waterflushing the PVA component. SA and PVDF-HFP were employed to match the RI of alcohol, and PVDF-HFP served as the shell layer to improve the hydrophobicity of the fibers. TEM images show that, with the SA concentration increased from $0 \%$ to $2 \%$, the interior structure evolved from a non-concentric structure to a core-shell structure. This evolution was also confirmed by XPS, where the intensity of $F_{1}$ increased and that of $\mathrm{O}_{1 \mathrm{~s}}$ decreased with increasing SA concentration. The formation of SA@PVDFHFP was associated with the competition between attractive forces from the Taylor cone, the incompatibility of the PVDF-HFP organic solvent phase and the PVA or SA/PVA aqueous phase, and internal stress produced by viscosity and surface tension. When the SA concentration was $1 \%$ or higher, the internal stress was enough to overcome the charge attractive forces and the high viscosity and surface tension 
weakened the asymmetric mobility of SA/PVA, leading to the formation of a core-shell structure observed. The as-prepared membranes exhibited extraordinary hydrophobicity, alcohol wettability with alcohol CA below $40^{\circ}$, and instantaneous light transmittance response with transparency exceeding 90\%. Besides, the as-prepared membrane could be reused for at least 10 cycles. The electrospun membrane was employed as a low-cost and rapidly-responsive smart packaging for visual detection of fruits freshness, which would effectively reduce food poisoning caused by improper food storage.

\section{AUTHOR INFORMATION}

\section{Corresponding Author}

*Fengwei Xie - School of Engineering, Newcastle University, Newcastle upon Tyne, NE1 7RU, United Kingdom; Email: fwhsieh@gmail.com

*Jiazi Hou - Key Laboratory of Automobile Materials of Ministry of Education, School of Materials Science and Engineering, Jilin University, Changchun 130025, China; Email: houjiazi@jlu.edu.cn

\section{Authors 604}

Yanan Xiao - Key Laboratory of Automobile Materials, Ministry Education, School of Materials Science and 606 Engineering, Jilin University, Changchun 130025, China

Hao Luo - Key Laboratory of Automobile Materials, Ministry Education, School of Materials Science and Engineering, Jilin 609 University, Changchun 130025, China 
Rongxing Tang - Key Laboratory of Automobile Materials, Ministry Education,

School of Materials Science and Engineering, Jilin University, Changchun 130025,

China

\section{Author Contributions}

Y X: Conceptualization, Methodology, Investigation, Data curation. H L: Data curation. R T: Date curation. F X: Supervision, Writing - Review \& Editing. J H: Conceptualization, Supervision, Formal analysis, Supervision, Resources, Writing review \& editing.

\section{Funding Sources}

The authors gratefully acknowledge financial support of this work by Jilin Province

Science and Technology Development Plan Project (202002011068JC).

\section{Notes}

The authors declare no competing financial interest.

\section{ACKNOWLEDGMENT}

The authors gratefully acknowledge the financial support for this work under a Jilin Province Science and Technology Development Plan Project (202002011068JC).

\section{REFERENCES}

1. Barbri, N. E.; Mirhisse, J.; Ionescu, R.; Bari, N. E.; Correig, X.; Bouchikhi, B.; Llobet, E., An Electronic Nose System Based on a Micro-Machined Gas Sensor Array to Assess the Freshness of Sardines. Sensors and Actuators B: Chemical 2009, 141 (2), 538-543. 2. Zhao, C.; Pan, Y.; Ma, L.; Tang, Z.; Zhao, G.; Wang, L., Assay of Fish Freshness Using Trimethylamine Vapor Probe Based on a Sensitive Membrane on Piezoelectric 
Quartz Crystal. Sensors and Actuators B: Chemical 2002, 81 (2-3), 218-222.

3. Chen, E. X.; Fu, H. R.; Lin, R.; Tan, Y. X.; Zhang, J., Highly Selective and Sensitive Trimethylamine Gas Sensor Based on Cobalt Imidazolate Framework Material. ACS Appl Mater Interfaces 2014, 6 (24), 22871-5.

4. Zhang, H.; Hou, A.; Xie, K.; Gao, A., Smart Color-Changing Paper Packaging Sensors with $\mathrm{pH}$ Sensitive Chromophores Based on Azo-Anthraquinone Reactive Dyes. Sensors and Actuators B: Chemical 2019, 286, 362-369.

5. Wang, Y.; Zhang, J.; Zhang, L., An Active and pH-Responsive Film Developed by Sodium Carboxymethyl Cellulose/Polyvinyl Alcohol Doped with Rose Anthocyanin Extracts. Food Chem 2021, 373 (Pt B), 131367.

6. Carbone, M.; Aneggi, E.; Figueredo, F.; Susmel, S., NiO-Nanoflowers Decorating a Plastic Electrode for the Non-Enzymatic Amperometric Detection of $\mathrm{H}_{2} \mathrm{O}_{2}$ in Milk: Old Issue, New Challenge. Food Control 2022, 132.

7. Dervisevic, M.; Custiuc, E.; Cevik, E.; Senel, M., Construction of Novel Xanthine Biosensor by Using Polymeric Mediator/MWCNT Nanocomposite Layer for Fish Freshness Detection. Food Chem 2015, 181, 277-83.

8. Rock, F.; Barsan, N.; Weimar, U., Electronic nose: current status and future trends. Chem Rev 2008, 108 (2), 705-25.

9. Guo, L.; Wang, T.; Wu, Z.; Wang, J.; Wang, M.; Cui, Z.; Ji, S.; Cai, J.; Xu, C.; Chen, X., Portable Food-Freshness Prediction Platform Based on Colorimetric Barcode Combinatorics and Deep Convolutional Neural Networks. Adv Mater 2020, 32 (45), e2004805.

10. Jia, R.; Tian, W.; Bai, H.; Zhang, J.; Wang, S.; Zhang, J., Amine-Responsive Cellulose-Based Ratiometric Fluorescent Materials for Real-Time and Visual Detection of Shrimp and Crab Freshness. Nat Commun 2019, 10 (1), 795.

11. Franco, M. R.; da Cunha, L. R.; Bianchi, R. F., Janus Principle Applied to Food Safety: An Active Two-Faced Indicator Label for Tracking Meat Freshness. Sensors and Actuators B: Chemical 2021, 333.

12. Siddiqui, J.; Taheri, M.; Alam, A. U.; Deen, M. J., Nanomaterials in Smart Packaging Applications: A Review. Small 2021, e2101171.

13. Cheng, H.; Xu, H.; Julian McClements, D.; Chen, L.; Jiao, A.; Tian, Y.; Miao, M.; Jin, Z., Recent Advances in Intelligent Food Packaging Materials: Principles, Preparation and Applications. Food Chem 2021, 375, 131738.

14. Varela, C., The Impact of Non-Saccharomyces Yeasts in the Production of Alcoholic Beverages. Appl Microbiol Biotechnol 2016, 100 (23), 9861-9874.

15. Zhang, T.; Gu, F.; Han, D.; Wang, Z.; Guo, G., Synthesis, Characterization and Alcohol-Sensing Properties of Rare Earth Doped $\mathrm{In}_{2} \mathrm{O}_{3}$ Hollow Spheres. Sensors and Actuators B: Chemical 2013, 177, 1180-1188.

16. Spagnoli, E.; Krik, S.; Fabbri, B.; Valt, M.; Ardit, M.; Gaiardo, A.; Vanzetti, L.; Della Ciana, M.; Cristino, V.; Vola, G.; Caramori, S.; Malagù, C.; Guidi, V., Development and Characterization of $\mathrm{WO}_{3}$ Nanoflakes for Selective Ethanol Sensing. Sensors and Actuators B: Chemical 2021, 347.

17. Wang, L.; Ma, S.; Li, J.; Wu, A.; Luo, D.; Yang, T.; Cao, P.; Ma, N.; Cai, Y., Mo- 
Doped $\mathrm{SnO}_{2}$ Nanotubes Sensor with Abundant Oxygen Vacancies for Ethanol Detection. Sensors and Actuators B: Chemical 2021, 347.

18. Giordano, G. F.; Freitas, V. M. S.; Schleder, G. R.; Santhiago, M.; Gobbi, A. L.; Lima, R. S., Bifunctional Metal Meshes Acting as a Semipermeable Membrane and Electrode for Sensitive Electrochemical Determination of Volatile Compounds. ACS Appl Mater Interfaces 2021, 13 (30), 35914-35923.

19. Shao, T.; Liu, L.; Li, Y.; Zhang, X.; Deng, Z.; Huo, L.; Gao, S., Highly Sensitive and Selective Detection of Long-Chain Alcohol Vapors Based on Keel-Type ZnO Fibers Derived from Waste Cigarette Butts. ACS Sustain Chem Eng 2021, 9 (17), 58385848 .

20. Bahuguna, G.; Mondal, I.; Verma, M.; Kumar, M.; Bhattacharya, S.; Gupta, R.; Kulkarni, G. U., Innovative Approach to Photo-Chemiresistive Sensing Technology: Surface-Fluorinated $\mathrm{SnO}_{2}$ for VOC Detection. ACS Appl Mater Interfaces 2020, 12 (33), $37320-37329$.

21. Kalytchuk, S.; Zdrazil, L.; Bad'ura, Z.; Medved, M.; Langer, M.; Paloncyova, M.; Zoppellaro, G.; Kershaw, S. V.; Rogach, A. L.; Otyepka, M.; Zboril, R., Carbon Dots Detect Water-to-Ice Phase Transition and Act as Alcohol Sensors via Fluorescence Turn-Off/On Mechanism. ACS Nano 2021, 15 (4), 6582-6593.

22. Yan, W.; Liu, Y.; Shao, G.; Zhu, K.; Cui, S.; Wang, W.; Shen, X., Chemical Surface Adsorption and Trace Detection of Alcohol Gas in Graphene Oxide-Based Acid-Etched $\mathrm{SnO}_{2}$ Aerogels. ACS Appl Mater Interfaces 2021, 13 (17), 20467-20478.

23. Torres-Costa, V.; Agulló-Rueda, F.; Martín-Palma, R. J.; Martínez-Duart, J. M., Porous silicon optical devices for sensing applications. Opt Mater 2005, 27 (5), 10841087.

24. Blackburn A, K. J. J., Adsorption from Binary Liquid Mixtures: Some Effects of Ash in Commercial Charcoal Journal of the Chemical Society (Resumed) 1955, 41034106.

25. Macdonald, E. K.; Shaver, M. P., Intrinsic high refractive index polymers. Polymer International 2015, 64 (1), 6-14.

26. Cai, M.; Yuan, D.; Zhang, X.; Pu, Y.; Liu, X.; He, H.; Zhang, L.; Ning, X., Lithium Ion Battery Separator with Improved Performance via Side-by-Side Bicomponent Electrospinning of PVDF-HFP/PI followed by 3D thermal crosslinking. J Power Sources 2020, 461.

27. Wang, Q.; Ju, J.; Tan, Y.; Hao, L.; Ma, Y.; Wu, Y.; Zhang, H.; Xia, Y.; Sui, K., Controlled Synthesis of Sodium Alginate Electrospun Nanofiber Membranes for MultiOccasion Adsorption and Separation of Methylene Blue. Carbohydr Polym 2019, 205, 125-134.

28. Bhattarai, R. S.; Bachu, R. D.; Boddu, S. H. S.; Bhaduri, S., Biomedical Applications of Electrospun Nanofibers: Drug and Nanoparticle Delivery. Pharmaceutics 2018, 11 (1).

29. Thompson, C. J.; Chase, G. G.; Yarin, A. L.; Reneker, D. H., Effects of Parameters on Nanofiber Diameter Determined from Electrospinning Model. Polymer 2007, 48 (23), 6913-6922. 
30. Zong, X.; Kim, K.; Fang, D.; Ran, S.; Hsiao, B. S.; Chu, B., Structure and Process Relationship of Electrospun Bioabsorbable Nanofiber Membranes. Polymer 2002, 43 (16), 4403-4412.

31. Sun, J. Y.; Zhao, X.; Illeperuma, W. R.; Chaudhuri, O.; Oh, K. H.; Mooney, D. J.; Vlassak, J. J.; Suo, Z., Highly Stretchable and Tough Hydrogels. Nature 2012, 489 (7414), 133-6.

32. Nassrullah, H.; Makanjuola, O.; Janajreh, I.; AlMarzooqi, F. A.; Hashaikeh, R., Incorporation of Nanosized LTL Zeolites in Dual-Layered PVDF-HFP/Cellulose Membrane for Enhanced Membrane Distillation Performance. J Membrane Sci 2020, 611 .

33. Tawansi, A.; Oraby, A. H.; Abdelrazek, E. M.; Abdelaziz, M., Structural and Electrical Properties of $\mathrm{MgCl}_{2}$-Filled PVDF Films. Polymer Testing 1999, 18 (8), 569579.

34. Chacko, S. K.; Rahul, M. T.; Raneesh, B.; Kalarikkal, N., Enhanced Magnetoelectric Coupling and Dielectric Constant in Flexible Ternary Composite Electrospun Fibers of PVDF-HFP Loaded with Nanoclay and $\mathrm{NiFe}_{2} \mathrm{O}_{4}$ Nanoparticles. New J Chem 2020, 44 (26), 11356-11364.

35. Daemi, H.; Barikani, M.; Barmar, M., Highly Stretchable Nanoalginate Based Polyurethane Elastomers. Carbohydr Polym 2013, 95 (2), 630-6.

36. Ahmad, A. L.; Farooqui, U. R.; Hamid, N. A., Effect of Graphene Oxide (GO) on Poly(vinylidene fluoride-hexafluoropropylene) (PVDF- HFP) Polymer Electrolyte Membrane. Polymer 2018, 142, 330-336.

37. Bonino, C. A.; Efimenko, K.; Jeong, S. I.; Krebs, M. D.; Alsberg, E.; Khan, S. A., Three-Dimensional Electrospun Alginate Nanofiber Mats via Tailored Charge Repulsions. Small 2012, 8 (12), 1928-36.

38. Wang, C.; Zuo, Q.; Wang, L.; Long, B.; Salleh, K. M.; Anuar, N. I. S.; Zakaria, S., Diameter Optimization of Polyvinyl Alcohol/Sodium Alginate Fiber Membranes Using Response Surface Methodology. Materials Chemistry and Physics 2021, 271.

39. Wang, H. C.; Li, Y.; Yang, M. J., Fast Response Thin Film $\mathrm{SnO}_{2}$ Gas Gensors Operating at Room Temperature. Sensors and Actuators B: Chemical 2006, 119 (2), 380-383.

40. Xu, X.; Li, C.; Pei, K.; Zhao, K.; Zhao, Z.; Li, H., Ionic Liquids Used as QCM Coating Materials for the Detection of Alcohols. Sensors and Actuators B: Chemical 2008, 134 (1), 258-265.

41. Kaushik, S.; Goswami, P., Bacterial Membrane Depolarization-Linked Fuel Cell Potential Burst as Signal for Selective Detection of Alcohol. ACS Appl Mater Interfaces 2018, 10 (22), 18630-18640.

42. Kim, J.; Jeerapan, I.; Imani, S.; Cho, T. N.; Bandodkar, A.; Cinti, S.; Mercier, P. P.; Wang, J., Noninvasive Alcohol Monitoring Using a Wearable Tattoo-Based Iontophoretic-Biosensing System. ACS Sensors 2016, 1 (8), 1011-1019.

43. Jiang, N.; Davies, S.; Jiao, Y.; Blyth, J.; Butt, H.; Montelongo, Y.; Yetisen, A. K., Doubly Photopolymerized Holographic Sensors. ACS Sensors 2021, 6 (3), 915-924.

44. Shankar, P.; Rayappan, J. B. B., Monomer: Design of ZnO Nanostructures 
(Nanobush and Nanowire) and Their Room-Temperature Ethanol Vapor Sensing Signatures. ACS Appl Mater Interfaces 2017, 9 (43), 38135-38145.

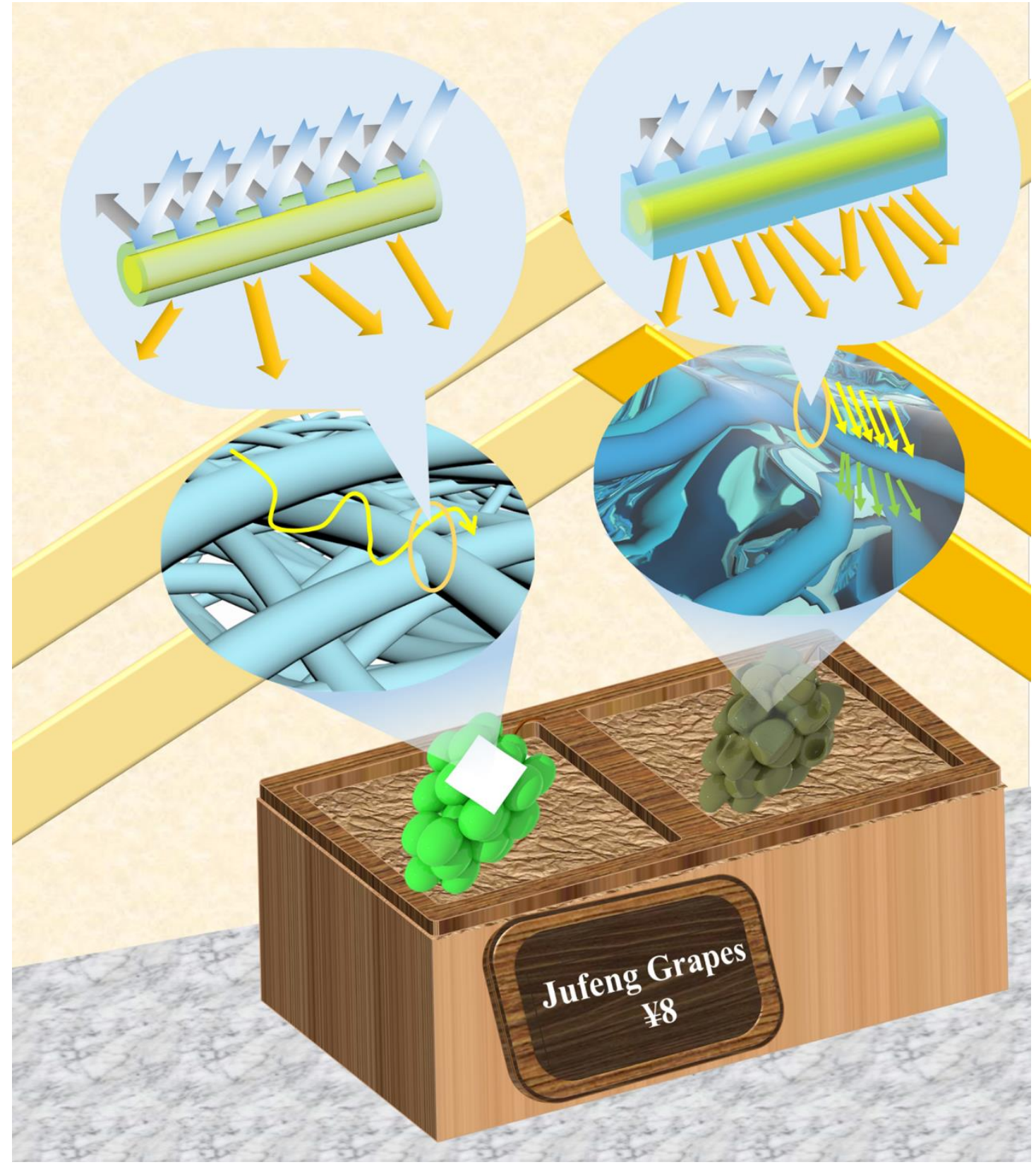

